\title{
Decision-making methods for operational flood management
}

\author{
K. A. Wojciechowska \\ HKV Consultants, The Netherlands
}

\begin{abstract}
In 1995, because of danger of flooding, a massive evacuation in the province of Gelderland in the Netherlands took place. The process of the evacuation went well, however, the life-threatening flood did not occur. The decision was based on deterministic information, experience and expertise. A robust technique, where uncertainties (e.g. in water level forecast) are explicitly incorporated, was not used (and is still not used). There are several methods in the decision theory, which allow the inclusion of uncertainty in decision-making in an explicit way. This paper gives a description of such methods in the context of operational flood management. We focus on decision trees, decision influence diagrams and Markov Decision Processes. In the context of operational flood management, the methods usually require specification of conditional flooding probability, i.e. a flooding probability given some (uncertain) information. We present application of such methods to operational decisions like evacuation or activation of an emergency storage area. We compare the methods with respect to ease of understanding and we briefly discuss whether there is a chance to apply the methods in real life situations.
\end{abstract}

Keywords: decision-making, decision trees, decision influence diagrams, Markov Decision Processes, uncertainty, conditional flooding probability.

\section{Introduction}

The Netherlands is a country located in the delta of three European great rivers: the Rhine, the Meuse and the Scheldt. Over $60 \%$ of the country is situated below mean sea level or is vulnerable to flooding by sea or by rivers. Natural barriers like dunes and high grounds, and man-made constructions like dikes and storm surge barriers protect the area from flooding. 
At the beginning of 1995, heavy precipitation in North France and the Ardennes led to rising of water levels on the rivers Meuse and Rhine. Consequently, two Dutch provinces, Limburg and Gelderland, were affected by the event. The situation in the province of Gelderland was especially dangerous, (Bezuyen et al. [1]). The condition of some dikes in the region was unsatisfactory. A breach in the dike system would cause flooding within a few hours in some valuable parts of the region. This fact and the resulting water depth could lead to life-threatening situations. The regional management team decided to evacuate part of the area. First, the evacuation was advised, shortly afterwards a call for compulsory evacuation was given (HWR [2]). The evacuation was massive, as almost 250,000 people were evacuated.

The process of evacuation went well, however, the life-threatening flooding did not occur. The evacuation decision was based mainly on deterministic information, experience and expertise (the reasons for the evacuation in 1995 were the water level forecasts, the duration of the high water levels and an assessment of the polder district that safety could not be assured due to the bad state of the dikes (Frieser [3])). Uncertainties in the information used (e.g. in water level forecasts) were not explicitly included in the decision-making process - a robust technique, where the uncertainties are explicitly incorporated, was not applied (and it is till now not in use).

The importance of uncertainties in water level forecast, during decisionmaking, can be explained on the basis of the Red River Flood of 1997. The flood was the result of high winter snow, river ice and unfavourable spring thaw. The event affected i.a. Grand Forks, a city in North Dakota (US). The water flowed far inland, leading to evacuation of the citizens (over 50,000 people were evacuated). In the face of potential flooding, the authorities did not decide to raise additional water defences because the maximal water level forecast did not go beyond prescribed safety levels (Verkade [4]). "The city engineer, relying on the 49 -foot $(14.5 \mathrm{~m})$ estimate by the National Weather Service, stated that, had they been told of the potential for a 54-foot $(16.5 \mathrm{~m})$ crest, they could have planned for and built a number of secondary dikes that would have saved much of the city from serious damage. If someone had told us that these estimates were not an exact science, or that other countries predict potential river crest heights in probabilities for various levels, we may have been better prepared." (Glassheim [5].)

There are several methods in the decision theory, which can be applied to include uncertainty in decision-making in an explicit way. This paper gives a description of such methods in the context of operational flood management. We focus on the decision trees, decision influence diagrams and Markov Decision Processes. In the context of operational flood management the methods usually require specification of conditional flooding probability defined as a flooding probability given some (uncertain) information. The importance of such probabilities has recently increased in the Netherlands. We present an application of such methods to operational decisions like evacuation or activation of an emergency storage area. We compare the methods with respect to ease of understanding (of importance for decision makers, who do not necessarily have a 
mathematical background) and we discuss whether there is a chance to apply the methods in real life situations.

\section{Emergency flood measures}

In this part two emergency flood measures will be considered. These measures are evacuation and activation of an emergency storage area. In the subsequent part we present the application of decision trees, decision influence diagrams and Markov Decision Processes; decisions about evacuation and the activation of an emergency storage area are modelled with help of the methods.

\subsection{Evacuation}

In this research, evacuation is defined as a movement of people away from a danger or occurrence of the danger, i.e. flood.

In general, two time aspects are involved with the evacuation decision, namely required and available time for evacuation (Barendregt et al [6]). The required time for evacuation consists of the time needed for decision-making (evacuation yes/no), preparation (initiation, warning and response) and transportation out of the area. The time available for evacuation is the period between the decision-making and the moment the calamity actually begins. Theoretically, if the time available for evacuation is larger than the time required for evacuation, a complete evacuation of inhabitants of the endangered area is possible. On the other hand, if the time available for evacuation is smaller than the time required for evacuation, a complete evacuation is not achievable prior to the occurrence of the flooding.

The primary goal of evacuation is to diminish (potential) flood consequences, so as to reduce the number of injured and fatalities. However, every evacuation entails costs. Initial evacuation costs, the value of direct and indirect economic damage contribute towards the costs, Frieser [3]. Initial evacuation costs contain e.g. compensations paid out to the evacuated people (in 1995, every evacuated household received about $€ 225$ from the Dutch government (Frieser [3]). Direct economic damage arises as a result of the suspension of production in the risk area and depends on the sort of economic branch (e.g. minerals extraction, banks/insurance companies, building industry) and duration of the suspension. Indirect economic damage consists of losses to supply companies and customers, and losses caused by problems in traffic in the regions outside the risk area.

\subsection{Emergency storage area}

An emergency storage area is a sparsely inhabited within-dikes area, which can be flooded in a controlled way, if extremely high river discharges occur (RBOS [7]). The aim of an emergency storage area is to decrease the peak of a high water wave and to consequently prevent flooding in within-dikes areas, which are situated downstream from the emergency storage area and have large socioeconomical importance. In short, an emergency storage area is an area flooded in 
favour of other area(s). The costs of activation of an emergency storage area resolved to damage to the area caused by the inflowing water.

Usability of an emergency storage area is restricted by volume of the area, meaning that the dike failure mechanism overflow is primary influenced by the controlled flooding. Influence of activation of an emergency storage area on other dike failure mechanisms (e.g. piping) is insignificant (RBOS [7]). In general, a human indirectly and directly determines the moment of activation of an emergency storage area. Indirectly, when an emergency storage area begins to work as soon as a fixed water level is exceeded, and directly, when an emergency storage area is activated by using a moveable inlet or by digging a breach. It is difficult to determine the best moment of activation of an emergency storage area, since such moment depends on partly an uncertain form of the discharge wave. It can happen that an emergency storage area is activated too early or too late. In Figure 1, two very theoretical examples are given: in the first and second panel correct and incorrect activation of an emergency storage area are depicted respectively (the modelling is done according to Nortier and de Koning [8]).

The continuous line in Fig. 1 represents a theoretical discharge wave at the Rhine (location Lobith; the river enters the Netherlands close to Lobith). The peak of the wave is equal to $17250 \mathrm{~m}^{3} / \mathrm{s}$; on average, this value is exceeded once per 3200 years at Lobith. The dashed line represents the discharge wave with a working emergency storage area. The area, with a volume of $161 \mathrm{mln} \mathrm{m}^{3}$, is
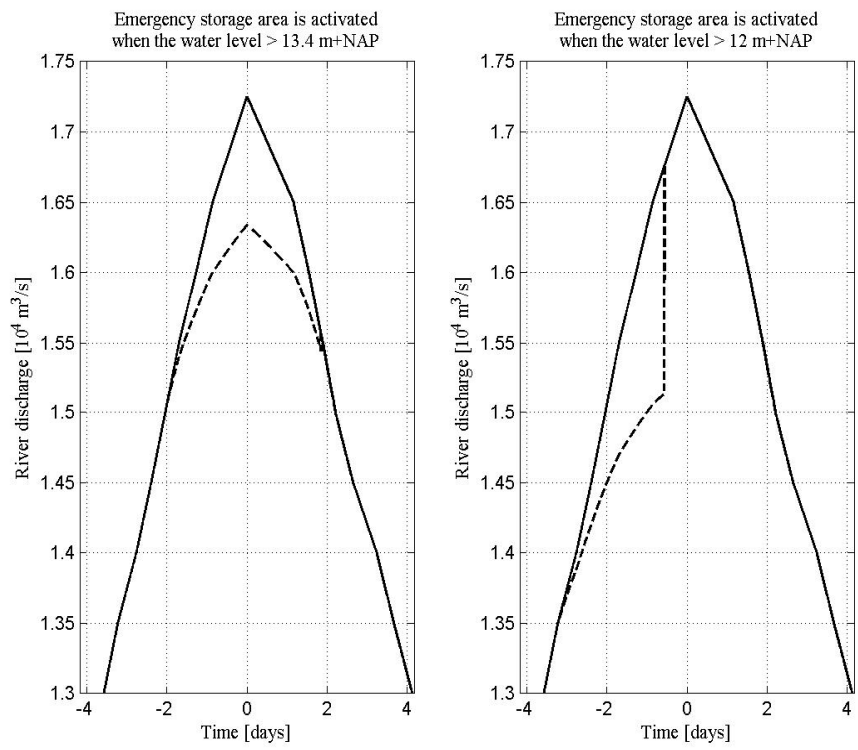

Figure 1: Influence of activation of an emergency storage area on the discharge wave. 
situated in the region. The measure is activated using a moveable inlet; it is elementarily assumed that the storage capacity of the area cannot be exceeded.

In the first panel of Figure 1, the area is activated when the water level at the location is higher than $13.4 \mathrm{~m}+\mathrm{NAP}$, leading to clear reduction of the peak. In the second panel, the area is activated when the water level exceeds $12 \mathrm{~m}+\mathrm{NAP}$. Such operation does not lead to reduction of the peak; in this situation the storage capacity of the area is used up before occurrence of the peak. In other words, the main danger (so the peak) was not diminished by the activation of the emergency storage area.

\section{Decision models}

In this part three mathematical decision models, often invoked in the decision theory, will be considered. These models are decision trees, decision influence diagrams and Markov Decision Processes. Both theory and application of the models will be presented.

\subsection{Decision trees}

\subsubsection{Definition}

Decision tree is a method, which helps in a decision-making involving high costs and probabilities of unwelcome events. The decision tree method uses a visual approach to compare considered decisions and to assign values to those decisions by combining probabilities and costs into specific values. In particular, these specific values are expected values.

A decision tree consists of branches and nodes. In general, the first node branches out into several branches, which represent decisions. Once a decision has been made one of several states of nature takes place; each state occurs with some probability. Furthermore, costs are associated with each decisions-state pair. The optimal decision is chosen on the basis of minimum expected costs criterion. More precisely, expected costs are determined for every decision and the decision with the lowest expected costs is indicated as the optimal decision. It is worth mentioning that in the case of a complicated decision problem, the corresponding decision tree usually has a very extensive form. For more theoretical information on the subject see Benjamin and Cornell [9].

\subsubsection{Application}

Let us consider application of the decision tree tool. Suppose that unfavourable weather conditions are forecasted. The forecasts are, however, uncertain and the uncertainty increases with time. The weather conditions can lead to flooding in a region. A decision maker, concerned about the weather forecast and condition of dikes in the region, considers at the present time evacuation of the region or activation of an emergency storage area (located upstream from the region). On the other hand, the decision maker knows that more exact information about the weather conditions will be available in the near future, hence eventual postponing of the decision is taken into account. Furthermore the decision maker 
is aware that the time aspect is fundamental in the case of the considered two flood measures.

A decision tree, proposed to model the situation, is shown in Figure 2 (a version of the tree is also given in Frieser [3]). The decision tree presents possible scenarios. One of the scenarios is the activation of the emergency storage area at time $t_{2}$ and no flooding at time $t_{0}$ (the lowest branch in Fig. 2). Another scenario is to postpone the decision problem till time $t_{1}$ (the "no action" arc in Fig. 2, starting in time $t_{2}$ and ending in time $t_{1}$ ). In the decision tree, costs (expressed as a pair: costs of a measure and the flood damage after taking the measure) are assigned to every scenario. The values are time dependent. Indeed, increase of the time available for evacuation leads to higher evacuation costs or later activation of an emergency storage area may result in lower flood damage. The model also contains information about the flooding probability at time $\mathrm{t}_{0}$ for a variety of circumstances. Probability $\mathrm{P}_{1}$ is defined as a flooding probability in the region at time $t_{0}$ given information available in time $t_{1}$. Probability $\mathrm{P}_{1} *$ is defined as a flooding probability in the region at time $t_{0}$ given information available in time $t_{1}$ and knowing that the emergency storage area has been activated (in contrast to an evacuation decision, activation of an emergency storage area has an influence on the flooding probability). Probabilities $\mathrm{P}_{2}$ and $\mathrm{P}_{2} *$ are defined analogically. These probabilities are by definition the conditional flooding probabilities. The available information can consist of water level forecasts and/or the expected condition of the dikes.

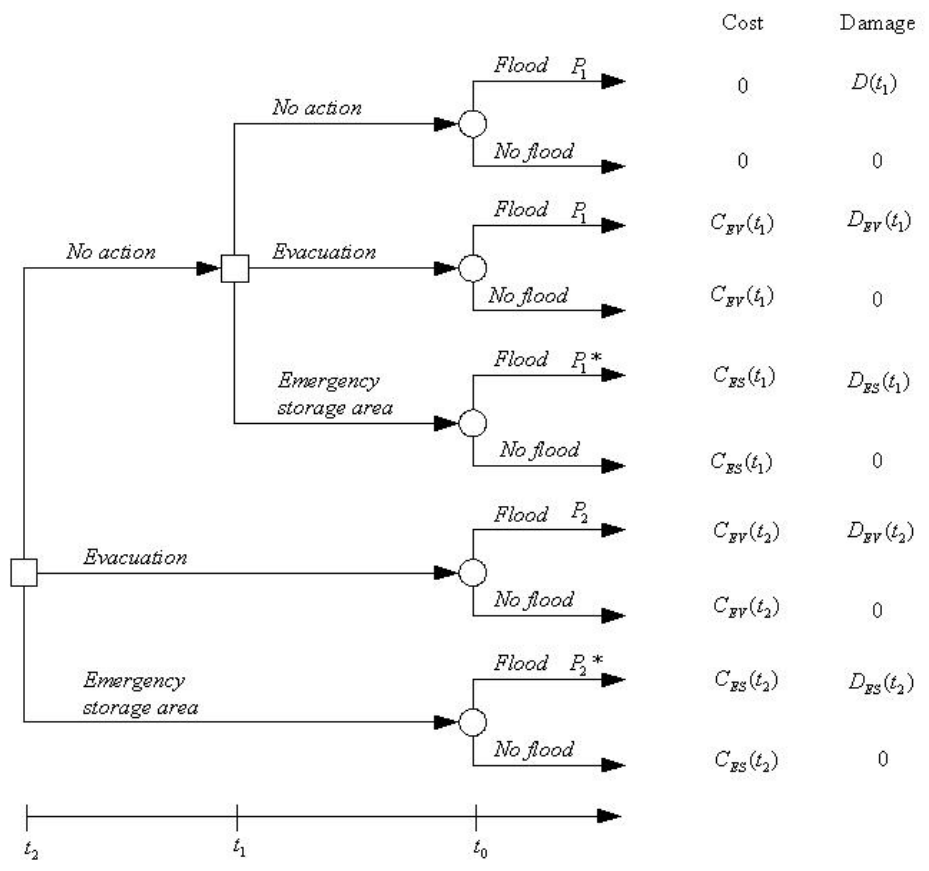

Figure 2: Application of the decision tree method. 
Let us emphasize, that the decision ("no action", "evacuation", "activation of an emergency storage area") has to be taken at time $t_{2}$, however, the decision tree model also requires specification of flooding probability at time $t_{0}$ given information available at time $t_{1}$ (note that time $t_{1}$ is assumed to be later than time $t_{2}$ ). In short, probability $P_{1}$ cannot be known at time $t_{2}$. The problem of estimating probability $\mathrm{P}_{1}$, being at time $\mathrm{t}_{2}$, has been tackled in the literature (see Frieser [3]). Estimation of probability $\mathrm{P}_{2}$ can be made as shown in Wojciechowska [10]. Estimation of probabilities $\mathrm{P}_{1}{ }^{*}$ and $\mathrm{P}_{2}{ }^{*}$ has not been discussed yet.

Estimation of costs and flood damage in the case of evacuation decision is in the most part presented in Frieser [3], whereas estimation of costs and flood damage in the case of activation of an emergency storage area is a subject for future work.

\subsection{Decision influence diagrams}

\subsubsection{Definition}

A decision influence diagram constitutes a compact graphical and mathematical representation of a decision problem, leading to better understanding and consequently to (rational) solving of the problem. In general, a decision influence diagram offers an intuitive way to identify and display the important elements of a decision-making process, including decisions, uncertainties and objectives, and relations (dependencies) between them.

More precisely, a decision influence diagram is defined as an acyclic directed graph (see Barlow [11]) where the following holds:

- Circle nodes represent random variables, double circle nodes denote deterministic values and squared nodes stand for decisions;

- Directed arcs into probabilistic and deterministic nodes show possible dependence, whereas directed arcs into decision nodes signifies available information at the moment of taking decision;

- A conditional probability (deterministic) function is associated with every probabilistic (deterministic) node, whereas a collection of decision rules is associated with every decision node (for more information on this subject see Barlow [11]);

- Decision nodes are in order; a decision node is (possibly implicitly) connected to all predecessor decision nodes;

- There exists exactly one deterministic node without any successors; such node is called the value node.

There are two operations used to solve decision problems represented by decision influence diagrams. These operations are the elimination of probabilistic nodes and the elimination of decision nodes (Barlow [11]). Decision influence diagrams are derived as an alternative to decision trees and the solution process of the both representations of a decision problem is fundamentally the same (Barlow [11]).

In general, a decision tree will have many more nodes and arcs than the corresponding decision influence diagram, what can make the decision tree method quite burdensome. Decision trees are, however, more useful in 
presenting all outcomes of decision problems. On the other hand, decision influence diagrams emphasize dependencies and independencies among variables more clearly than the related decision tree.

\subsubsection{Application}

In Figure 3, a decision influence diagram is presented. The diagram corresponds to the decision problem described in paragraph 3.1.2. In other words, the diagram is an alternative to the decision tree shown in Fig. 2.

Let us consider the diagram. The diagram consists of four nodes, each with prescribed meaning. Moreover, five directed arcs are contained in the diagram. As already mentioned, the arcs indicate possible dependence. All considered decisions are represented by a one squared node. Random variability of the flood event at time $t_{0}$ is represented by a single circle node. Available information (known during the decision-making and essential for assessing the flooding probability) and values corresponding to the decision-state pairs are represented by double circles. The decisions and (uncertain) flood event have an influence on the inquired costs therefore the corresponding nodes are connected. Taken decision can have an influence on the flooding probability at time $t_{0}$, thus, a directed arc from the decision node to the random node has been drawn (e.g. activation of the emergency storage area has an influence on the flooding probability).

Clearly, the diagram has a much more compact form than the corresponding decision tree leading to better visibility of present variables and connections between them.

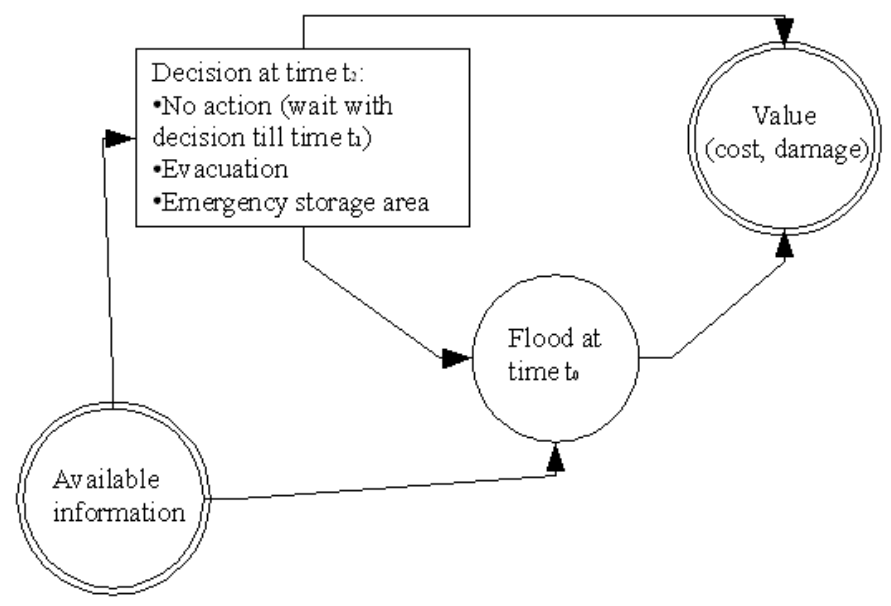

Figure 3: Application of the decision influence diagram method (alternative to Fig. 2). 


\subsection{Markov Decision Processes}

\subsubsection{Definition}

Markov Decision Processes offer a mathematical structure for modelling of a decision-making in circumstances when results are partly random and partly under the influence of a decision maker.

A Markov Decision Process is a stochastic process observed at discrete time points. At every time point the process is found in one of possible states. After observing the state of the process, an action is taken. When the process is in state $i$ at time $t$ and action a is chosen, then (bounded) cost $C(i, a)$ is incurred and the next state of the system, say $j$, is chosen with probability $P_{i j}(a)$, hence $P_{i j}(a)$ is a transition probability from state $i$ to state $j$ when action $a$ is taken. Both the costs and the transition probabilities are functions only of the last state and the upcoming action. Moreover, it is assumed that the number of all actions is finite.

To choose an action (depending on a state), a decision maker must act according to some policy. A policy can be simply a function from the state space into the action space. In the case of short-term decisions (measured in days), the aim is to determine an average cost optimal policy, which minimizes average expected cost for all initial states. In general, the optimal policy can be identified using algorithms proposed in the literature (e.g. value iteration algorithm or policy improvement algorithm). For more theoretical information on the subject see Ross [12].

\subsubsection{Application}

Let us consider application of the Markov Decision Processes to operational flood management. For the sake of simplicity, only evacuation decision will be considered. It is assumed that a system consists of monetary consequences of (eventual) flooding and actual flooding probability. The latter is defined as a flooding probability given actual information. Further, it is assumed that the system can be found in one of five states. State 0 corresponds to an ideal situation, where flooding danger is very small (the consequences-probability pair is not specified for this state). It is assumed that state 0 is an absorbing state (it is not possible to leave this state) and that the system cannot start in this state. State 1 corresponds to the situation where the consequences of (eventual) flooding are equal to $\mathrm{C}$ and the actual flooding probability is equal to $\mathrm{P}_{1}$, this is written as $1=\left\{C, P_{1}\right\}$. Similarly for the three following states, we define $2=\left\{C, P_{2}\right\}, 3=\left\{C_{1}\right.$, $\left.\mathrm{P}_{1}\right\}$ and $4=\left\{\mathrm{C}_{1}, \mathrm{P}_{2}\right\}$, where $\mathrm{C}_{1}<\mathrm{C}$ and $\mathrm{P}_{1}<\mathrm{P}_{2}$.

In the example two actions lead to transition between the states: evacuation and no evacuation (no action). In states 1 and 2 the two actions are possible, whereas in states 0,3 and 4 only the latter action can be chosen.

Let us now consider the system being in state 1 at the present time point. No action can lead to state 0 (ideal situation) or to state 2 (increase of flooding probability) at the next time point, but there is also a possibility that the state of the system will not change at the following time point. Evacuation can lead to states 0,3 or 4 at the next time point. In state 3 the consequences of flooding are smaller $\left(\mathrm{C}_{1}<\mathrm{C}\right)$ and the flooding probability does not change. It is reasonable that consequences in state 3 are less severe, because evacuation reduces potential 
flood damage. In state 4 the consequences of flooding are less severe, but the flooding probability increases. In states 3 and 4 no action is possible, leading to absorbing state 0 . Similar considerations can be made for the system being in state 2 at the present time point, entailing Figure 4.

For a specific action, probabilities affect transitions between the states. Let us remark, that e.g. the probability of transition from state 2 to state 1 , when no action is taken, is equal to the probability of transition from state 2 to state 3 when evacuation is chosen. Also, according to the principle of the method, costs are associated with every taken action. In the considered problem the costs will be additionally dependent on the following state and can be defined as the effectiveness of the chosen action (the costs will be in fact rewards). The aim of the model is to determine the best action being in state 1 or 2 (note that for the other states the "best" actions are already specified).

Let us emphasize that the presented application of Markov Decision Processes is rather fake (it is created for the purpose of this paper) and does not correspond to the classical book examples, where usually all actions are available in every state of a system (see Ross [12]). An evacuation decision can be made once; allowing the evacuation decision to be chosen for the first at time $t$ and then again at time $\mathrm{t}+2$ could lead to a paradox. The same observation holds for a Markov Decision Process, where activation of an emergency storage are is one of possible actions.

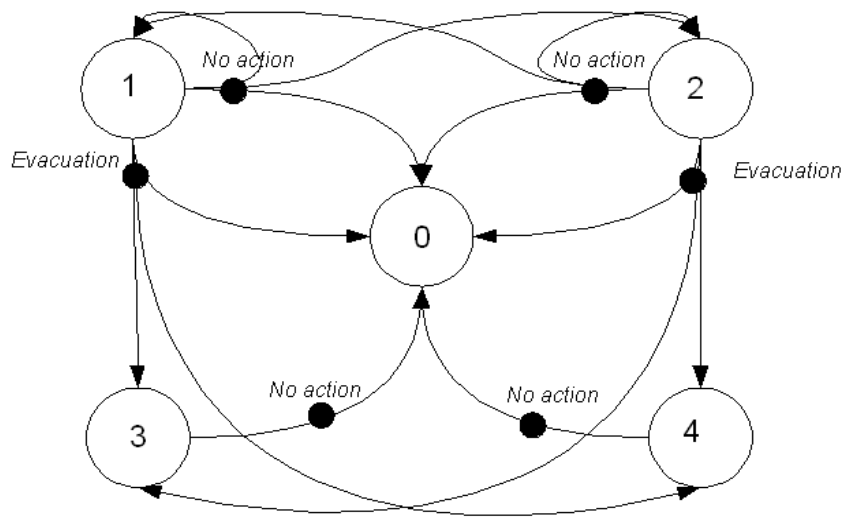

Figure 4: Application of the Markov Decision Processes.

\section{Discussion, conclusions and future work}

Discussion, conclusions and future work following from this research can be summarised as follows:

- As it has been already mentioned, decision trees and decision influence diagrams are alternatives - despite all differences, the solution process in both methods is fundamentally the same. We state to determine both 
decision tree and decision influence diagram for a decision problem, since the graphs (visually) complete each other leading to a better understanding of the problem.

- In contrast to Markov Decision Processes, the application of decision trees and decision influence diagrams is rather intuitive and does not require extensive mathematical knowledge. In general, a Markov Decision Process cannot be translated to a decision tree or a decision influence diagram - the methods tackle slightly different decision problems.

- In the presented application of the Markov Decision Processes, several parameters have to be specified (e.g. transition probabilities, probabilities $P_{1}$ and $\mathrm{P}_{2}$ ). A large number of unknown parameters can constitute a difficulty during crisis situations.

- The presented application of Markov Decision Processes is rather fake and does not correspond to the classical book examples, where usually all actions are available in every possible state of a system (see Ross [12]). Evacuation decision (or, e.g., activation of an emergency storage are) can be made once; allowing an evacuation decision to be chosen for the first at time $t$ and then again at time $t+2$ could lead to a paradox.

- The considered decision methods allow one to include mathematically some uncertainties. It is important to emphasize that the methods can be applied as a rational means of support in real-life situations - the methods cannot replace the usual decision-making, where often intuition or other information is used. Furthermore, the methods constitute rather a schematic approach to decision problems.

- The considered decision methods are not applied in operational flood management in the Netherlands. Introduction of the methods would certainly require time. Both developing and learning processes should be a common work of scientists and end-users (so decision makers); this could prevent a gap between the scientists' expectations and the end-users' needs.

- Future work concerning the subject should focus on the estimation of the required parameters i.e. conditional flooding probabilities $\mathrm{P}_{1}{ }^{*}$ and $\mathrm{P}_{2}{ }^{*}$, costs and flood damage in the case of activation of an emergency storage area after (in the decision tree) or the transition probabilities (in the Markov Decision Process).

\section{Acknowledgement}

This research is part of the Flood Control 2015 program. The program is partly funded by the Dutch Ministry of Transport, Public Works and Water Management. For more information visit http://www.floodcontrol2015.com.

\section{References}

[1] Bezuyen, M.J., van Duin, M.J. \& Leenders, P.H.J.A., Flood Management in The Netherlands, Australian Journal of Emergency Management, winter 1998. 
[2] HWR, Hoog Water 1995, Regio Nijmegen, Evaluatierapport (in Dutch), 1995.

[3] Frieser, B.I., Probabilistic Evacuation Decision Model for River Floods in the Netherlands, Master's thesis, Delft University of Technology and Ministry of Transport, Public Works and Water Management, Delft, June 2004.

[4] Verkade, J., The value of flood warning systems, M.Sc. dissertation, Water resources Management programme Delft University of Technology, Delft, June 2008.

[5] Glassheim, E., Fear and loathing in North Dakota, Natural Hazards Observer, XXI, 1-4, 1997.

[6] Barendregt, A., van Noortwijk, J.M., van der Doef, M. \& Holterman, S.R., Determining the time available for evacuation of a dike-ring area by expert judgement, ISSH - Stochastic Hydraulics 2005 - 23 and 25 May 2005 Nijmegen, the Netherlands.

[7] RBOS, Rampenbeheersingsstrategie Overstromingen Rijn en Maas Achtergrondrapportage Veiligheid en rivierkunde (in Dutch), RIZA report 2005.024, Ministerie van Verkeer en Waterstaat, December 2005.

[8] Nortier, I.W. \& de Koning, P., Toegepaste Vloeistofmechanica, Hydraulica voor waterbouwkundigen (in Dutch), Chapter 7. Stroming over overlaten en meetschotten. Stuwen. Afvoercoefficient. Wolters-Noordhoff bv Groningen/Houten, The Netherlands, pp. 278-279, 1996.

[9] Benjamin, J.R. \& Cornell, C.A., Probability Statistics and Decision for Civil Engineers, Chapter 5. Elementary Bayesian Decision Theory, McGraw-Hill, Inc., pp. 526-581, 1970.

[10] Wojciechowska, K.A., Extension of the probabilistic evacuation decision model, Proc. of the $7^{\text {th }}$ International Probabilistic Workshop 25-26 November, eds. P. van Gelder, D. Proske \& H. Vrijling, Delft, the Netherlands, pp. 313-325, 2009.

[11] Barlow, R.E., Engineering Reliability, (Chapter 10). Making Decisions Using Influence Diagrams, University of California, Berkeley, the American Statistical Association and the Society for Industrial and Applied Mathematics, pp. 165-181, 1998.

[12] Ross, S.M., Applied Probability Models with Optimization Applications, (Chapter 6). Markov Decision Processes, Dover Publications INC., New York, pp. 119-152, 1970. 